\title{
JAK-ing up inadequate RA therapy
}

For patients with rheumatoid arthritis (RA) that was active despite previous methotrexate therapy, the combination of tofacitinib plus methotrexate was as effective as adalimumab plus methotrexate in a head-to-head trial. However, tofacitinib monotherapy did not show non-inferiority to either combination therapy. "These results would suggest that in a group of patients who had an incomplete response to methotrexate, but who can tolerate methotrextate, it would be better to first add tofacitinib rather than to

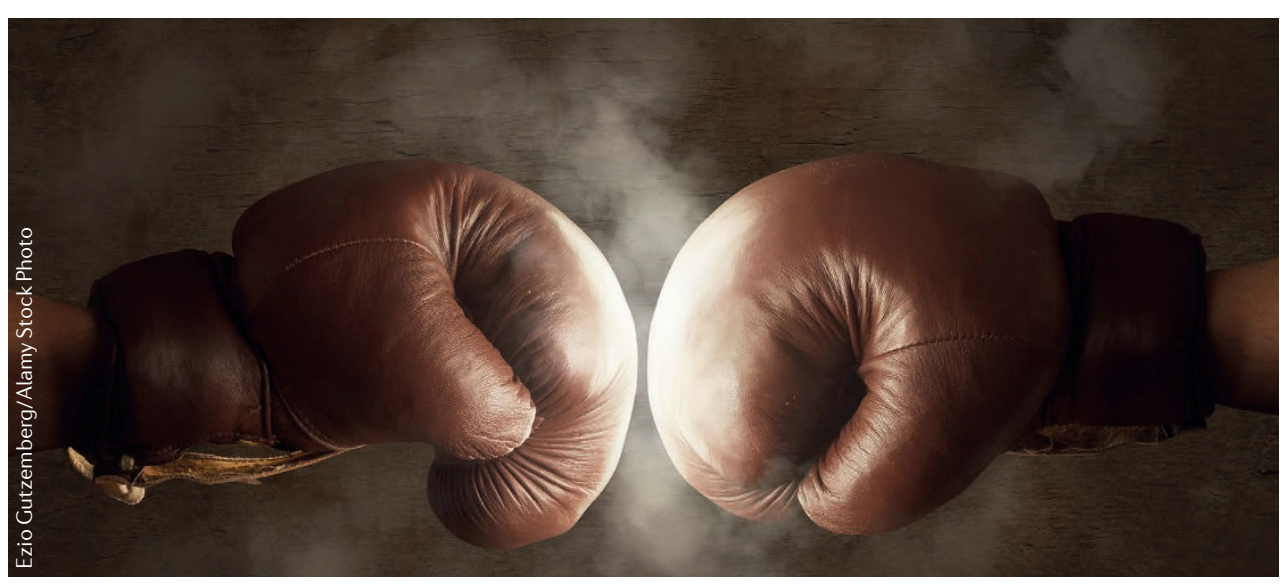

switch to tofacitinib," explains corresponding author Roy Fleischmann.

The study, called ORAL Strategy, was a 1 -year, phase $3 \mathrm{~b} / 4$ randomized controlled trial that included patients at 194 centres in 25 countries. At 6 months, $46.0 \%$ ( $n=173)$ of patients who were treated with the orally administered Janus kinase (JAK) inhibitor tofacitinib (5 mg twice daily) plus methotrexate achieved an ACR50 response, comparable to the $43.8 \%(n=169)$ of those treated with adalimumab (40 mg every other week) plus methorexate ...tofacitinib plus methotrexate was as effective as adalimumab plus methotrexate in a head-to-head trial

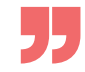

who achieved this primary end point. Among those who received tofacitinib monotherapy (5 mg twice daily), 38.3\% ( $n=147)$ achieved an ACR50 response at 6 months. Rates of therapy discontinuation due to adverse events were similar across all three treatment groups.

Clinical trials are underway to assess whether methotrexate therapy could be withdrawn after a state of low disease activity has been achieved with the combination of tofacitinib and methotrexate. "If a patient does well with the combination," says Fleischmann, "then an attempt can be made to taper or discontinue methotrexate, with an expectation that a majority of patients will be able to do so."

Sarah Onuora

ORIGINAL ARTICLE Fleischmann, R. et al.

Efficacy and safety of tofacitinib monotherapy, tofacitinib with methotrexate, and adalimumab with methotrexate in patients with rheumatoid arthritis (ORAL Strategy): a phase $3 \mathrm{~b} / 4$, doubleblind, head-to-head, randomised controlled trial. Lancet http://dx.doi.org/10.1016/S0140-

6736(17)31618-5 (2017)

FURTHER READING Schwartz, D. M. et al. Type I/II cytokines, JAKs, and new strategies for treating autoimmune diseases. Nat. Rev. Rheumatol. 12, 25-36 (2016) 